\title{
New E. coli vaccine covers more bases
}

- Nicola Moreland -

Oral Escherichia coli vaccine [ETEC/rCTB] reduces the incidence of moderate-to-severe enterotoxigenic $E$. coli (ETEC) illness and the severity of ETEC illness in travellers, according to a large study presented at the 45th Interscience Conference of Antimicrobial Agents and Chemotherapy (ICAAC) [Washington, DC, US; December 2005]. This double-blind, randomised study included 1458 US adults travelling to Guatemala and Mexico who received two doses of vaccine or placebo approximately 3 weeks and 1 week before departure. Among subjects with a strong immune response, protective efficacy was $84 \%$ against moderate-to-severe ETECassociated traveller's diarrhoea. Furthermore, traveller's diarrhoea caused by ETEC was significantly milder in the vaccine group than in placebo recipients.

Traveller's diarrhoea is the primary cause of illness in international travellers to developing countries; approximately $30-50 \%$ of travellers from industrialised countries going to high-risk areas may experience at least one episode of traveller's diarrhoea during their trip. ETEC is estimated to cause between $22-60 \%$ of all cases of traveller's diarrhoea.

Acquired through the consumption of contaminated food, ETEC causes diarrhoea by sticking to the GI tract surface via colonisation factor antigens (CFA), and produces heat-sensitive toxin (LT), heat-stable toxin (ST), or both. ETEC-associated traveller's diarrhoea is generally not life-threatening; however, around $40 \%$ of moderate-to-severe cases cause disruptions in travel activities and about $30 \%$ seek medical care or stay in their rooms.

Presently, an oral, inactivated whole cell cholera vaccine, supplemented with the B-subunit of cholera enterotoxin (CTB) [Dukoral], is the only vaccine available providing some protection against ETEC; this vaccine offers only short-term protection against LT toxin-producing strains.

\section{New ETEC vaccine}

Therefore, an ETEC vaccine with protection against both LT toxin and CFA-expressing ST toxin-producing strains would increase the range of vaccine protection and significantly improve prophylaxis options for travellers. A new oral vaccine currently undergoing development consists of formulin-killed E. coli whole cells expressing common CFAs plus CTB, and is designed to induce immunity against ETEC strains expressing the LT toxin in addition to strains expressing the most common CFA antigens.

In a previous trial, an earlier prototype of the $E$. coli vaccine provided protective immunity against more severe traveller's diarrhoea caused by ETEC strains sharing toxin or CFA antigens with the vaccine (protective efficacy $77 \% ; p=0.03$ ). A larger study, presented at the ICAAC meeting, was conducted to further evaluate the efficacy and tolerability of this $E$. coli vaccine in US travellers visiting Guatemala and Mexico. ${ }^{1}$

In this double-blind study, 1458 adult volunteers were recruited who were intending to travel to Guatemala and Mexico. They were randomised to receive two doses of the $E$. coli vaccine or placebo approximately 3 weeks and 1 week prior to departure, respectively. The oral vaccine or placebo was self-administered at home with supervision via telephone.

Subjects were then followed-up for 14-28 days into their travel. Routine serum and faecal samples were collected at days 0,7 and 14-21 days and additional stool samples were collected during illness. Faecal samples from diarrhoea cases were tested for ETEC strains sharing vaccine CFAs.
The per-protocol population consisted of 1406 subjects who completed surveillance, including 705 vaccinees and 701 placebo recipients. Baseline characteristics were comparable across treatment groups.

On arrival, 517 study participants, including 322 vaccinees, had serum IgA titres against CTB, as determined by ELISA, with a median anti-CTB IgA titre of 1358.

Among 59 E. coli vaccine recipients, serum IgA response rates were almost $90 \%$ for $\mathrm{CTB}$, over $10 \%$ for CFA/I and almost $20 \%$ for CS1 strains. The respective rates for 57 placebo recipients ranged from 0 to $5 \%$.

\section{Encouraging results}

The primary study endpoint was cases of traveller's diarrhoea (five loose watery stools in 24 hours and Gl symptoms) caused by ETEC sharing vaccine antigens, with no other pathogen isolated. Secondary outcomes included the incidence of moderate-to-severe ETEC disease, defined as diarrhoea plus gastrointestinal symptoms interfering with daily activity.

Self-administered $E$. coli vaccine was shown to be safe and immunogenic in this subject population. The overall incidence of traveller's diarrhoea was 34\% in Guatemala and $23 \%$ in Mexico. About half of these cases were reported as moderate-to-severe ETEC illness.

\begin{tabular}{|l|}
\hline $\begin{array}{l}\text { Protective efficacy against ETEC illness in vaccine } \\
\text { recipients with anti-CTB titres of } \geq \mathbf{1 3 5 8}\end{array}$ \\
\hline \multicolumn{2}{|c|}{$\begin{array}{c}\text { Vaccine efficacy rate vs } \\
\text { placebo }\end{array}$} \\
\hline $\begin{array}{l}\text { ETEC-associated traveller's diarrhoea: } \\
\text { Gl symptoms of any intensity }\end{array}$ \\
$\begin{array}{l}\text { Gl symptoms interfering with daily } \\
\text { activity }\end{array}$ \\
$\begin{array}{l}\text { Most severe Gl symptoms } \\
* p<0.05\end{array}$ \\
$* * p<0.01$ \\
\hline
\end{tabular}

Moderate-to-severe ETEC was reported in fewer vaccine recipients than placebo recipients, although the difference was not statistically significant (4/705 vs 10/701). However, protection from traveller's diarrhoea caused by ETEC was greater in subjects with stronger immune responses following vaccination.

Among participants with an anti-CTB titre of $\geq 1358$, protective efficacy of the vaccine was $84 \%(p=0.004)$ against moderate-to-severe ETEC illness and 63\% $(\mathrm{p}=0.02)$ against milder ETEC traveller's diarrhoea, compared with placebo [see table]. The vaccine provided $100 \%$ protection from ETEC-associated traveller's diarrhoea with most severe symptoms ( $p=0.04$ vs placebo). Overall, traveller's diarrhoea 


\section{New E. coli vaccine covers more bases - continued}

caused by ETEC was milder in vaccine recipients with a strong immune response than in placebo recipients ( $p \leq 0.04)$. Furthermore, cases reported in vaccine recipients with stronger immune responses were of significantly shorter duration and caused significantly less change in daily activity, compared to placebo.

The $E$. coli vaccine was well tolerated. Transient mild nausea and abdominal pain or cramps after dosing were observed in both study groups at a rate of $10-12 \%$.
1. Bourgeois AL, et al. Protective efficacy of an oral killed vaccine for enterotoxigenic E. coli in adult travelers to Guatemala and Mexico. 45th Interscience Conference on Antimicrobial Agents and Chemotherapy : 234 (plus oral presentation) abstr. G-408, Dec 2005.

800999872

》 Editorial comment: The oral E. coli vaccine [SBL Vaccin AB] is in phase III studies in Guatemala, Kenya and Mexico for the prevention of E. coli infections. 